\title{
Top quark pair and single top t-channel differential cross sections in CMS
}

\author{
Youn Jung Roh*Korea University (KR) \\ E-mail: youn.jung.rohecern.ch \\ on behalf of the CMS Collaboration
}

\begin{abstract}
Differential measurements of top quark pair and single top quark (t-channel) production cross sections are presented using data collected by CMS at different center-of-mass energies. The cross sections are measured as a function of various kinematic observables of the top quarks and the jets and leptons of the event final state. The $t \bar{t}$ measurements are extended to the TeV range using jet substructure techniques to exploit the boosted regime. The results are confronted with precise theory calculations.
\end{abstract}

XXVI International Workshop on Deep Inelastic Scattering and Related Subjects 16-20 Apr 2018

Kobe, Japan

${ }^{*}$ Speaker. 


\section{Introduction}

Measurements of top quark pair and single top quark cross section can test the standard model (SM) and probe new physics. The top quark process is an important background of many searches and other measurements. Precise measurements allow improving the modeling of top quark processes. It helps in validating the perturbative QCD calculations and better understanding the parton distribution function (PDF).

The CMS collaboration [1] has published many top pairs and single top quark measurements $[2,3,4,5,6,7,8,9,10]$ in different final states such as dilepton, semi-lepton, and all jets channels at center-of-mass energies of 8 and $13 \mathrm{TeV}$. The measurements are performed as a function of various kinematic observables. The $t \bar{t}$ measurements extended to the $\mathrm{TeV}$ range are carried by jet substructure techniques to investigate the boosted regime. In this note, the recent measurements of differential $t \bar{t}$ and t-channel single top cross sections and double-differential $t \bar{t}$ cross section are described.

\section{Differential $t \bar{t}$ cross sections in the dilepton channel}

The normalized differential cross sections at both particle and parton levels are measured at 8 and $13 \mathrm{TeV}$. The measurements are performed at the particle level, using final state objects defined in a theoretically safe and unambiguous way. Precise measurements are unfolded to parton and particle level in order to compare with high order QCD calculations. As shown in Figure 1, top quark $p_{T}$ distributions are softer in data than the predictions both at 8 and $13 \mathrm{TeV}$. In general, data measurements are well described by the SM predictions.
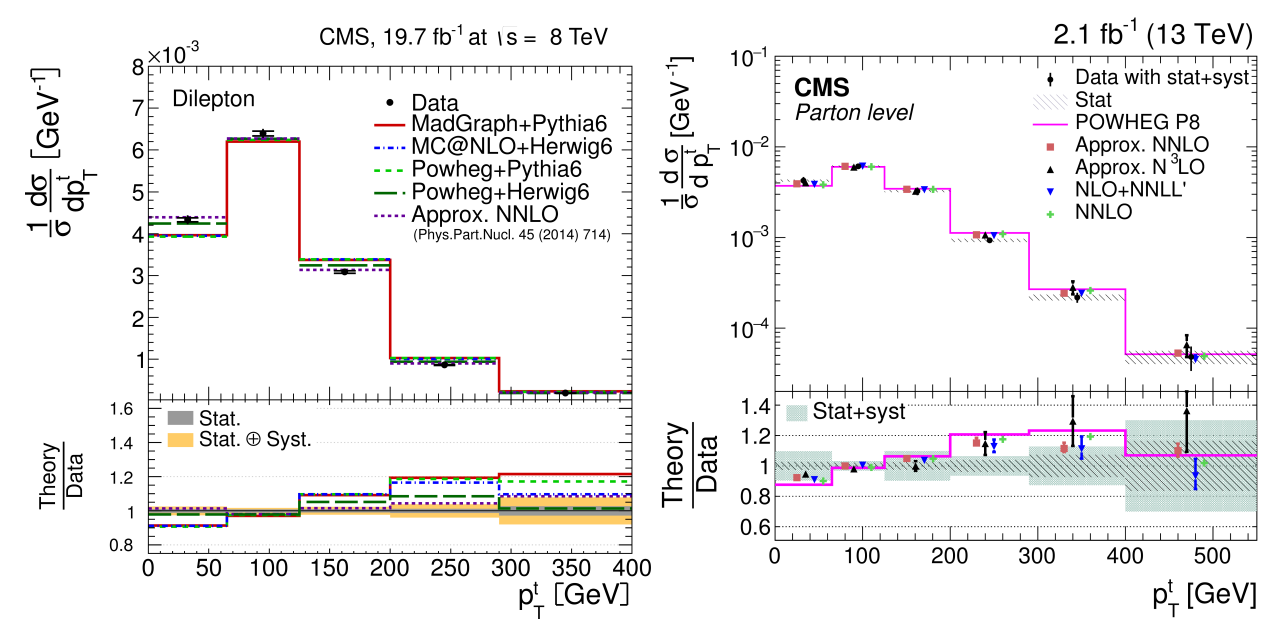

Figure 1: The error bars on data indicate total (combined statistical and systematic) uncertainties. The dark shaded band (left) and the hatched band (right) shows the statistical uncertainty. The measurements are compared to different perturbative QCD calculations $[2,3]$. 


\section{Differential $t \bar{t}$ cross sections in the semi-leptonic channel}

The measurements of differential cross sections in the semi-leptonic channel are performed at 8 and $13 \mathrm{TeV}[4,5,6]$. Differential cross sections at parton level are measured as function of top quark $p_{T}$ and compared to the available predictions. As shown in Figure 2 (left), they are also softer in data than the predictions, and the measurements agree better with the NNLO QCD + NLO EW calculation. The boosted regime is also measured as shown in Figure 2 (right). Generally, data measurements are found to be well described by the SM predictions.
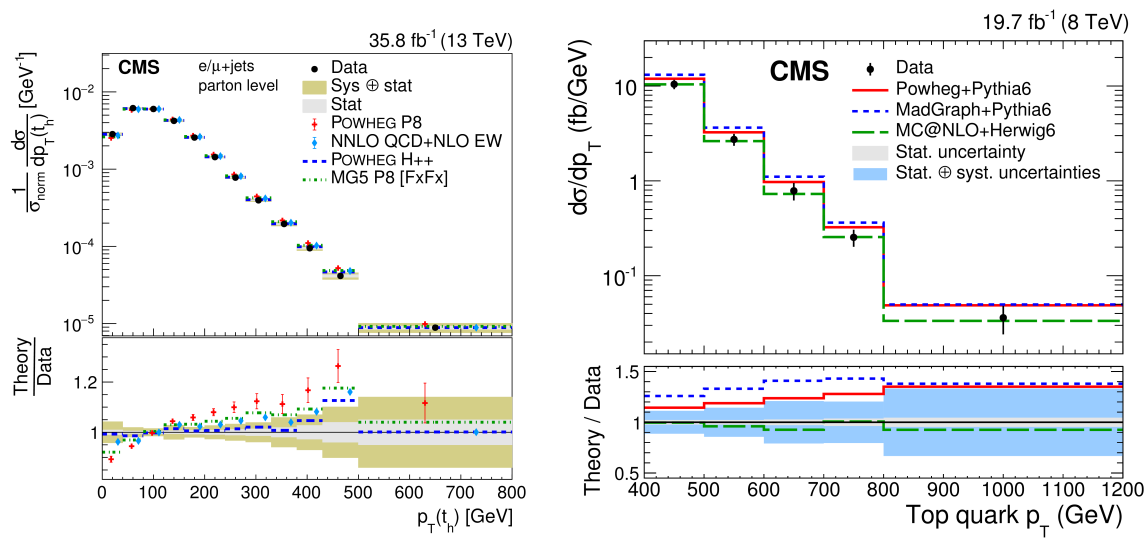

Figure 2: The error bars on data indicate total (combined statistical and systematic) uncertainties. The dark shaded band shows the statistical uncertainty. The measurements are compared to different theory predictions $[5,6]$. The boosted regime is also investigated (right).

\section{Differential $t \bar{t}$ cross sections in the all jets channel}

Differential $t \bar{t}$ cross section in all jets channel at 8 and $13 \mathrm{TeV}$ are measured in the resolved and boosted regime $[7,8]$. The measurements are extended to the $\mathrm{TeV}$ range using jet substructure techniques to investigate the boosted regime. Figure 3 shows the $t \bar{t}$ cross section as a function of the leading top quark $p_{T}$ at parton level in the boosted (left) and detector level in the resolved and boosted regime (right). The measurements are compared to different theory predictions.

\section{Double-differential $t \bar{t}$ cross sections}

Double-differential $t \bar{t}$ cross sections can disentangle effects and set constraints on PDFs. The measurements of double-differential $t \bar{t}$ cross section are performed in the dilepton and semi-leptonic channel at 8 and $13 \mathrm{TeV}[9,6]$. The measurements at $8 \mathrm{TeV}$ are compared to the NLO (MNR) prediction calculated with CT14 and HERAPDF2.0 and the approximate NNLO (DiffTop) prediction calculated with CT14. The double-differential $t \bar{t}$ cross sections at $13 \mathrm{TeV}$ are measured at parton and particle level and compared to the predictions. Data measurements are found to be in good agreement with the SM predictions as shown in Figure 4 . 

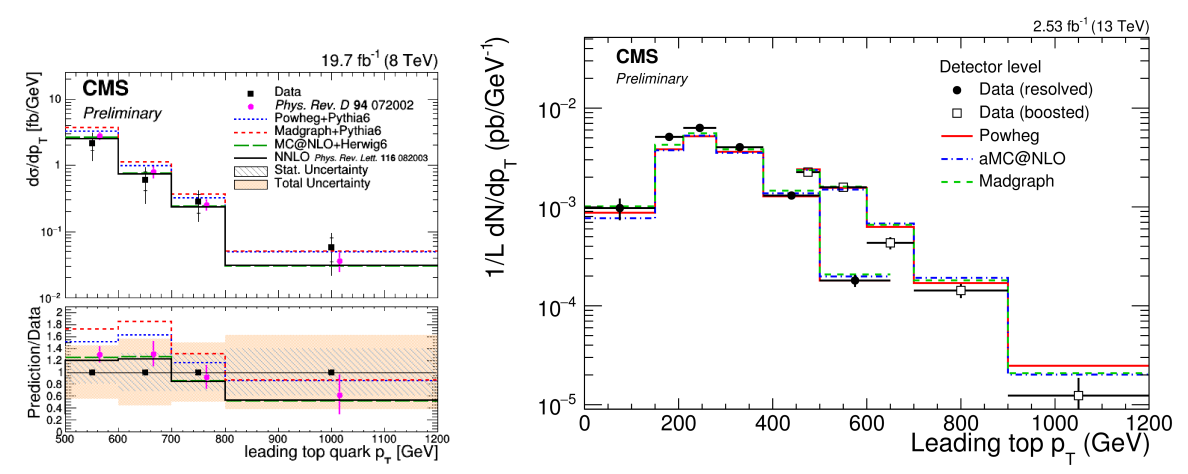

Figure 3: Differential $t \bar{t}$ cross section in all jets channel at 8 and $13 \mathrm{TeV}$ are measured as a function of the leading top quark $p_{T}$ at parton level in the boosted (left) and detector level in the resolved and boosted regime (right). The measurements are compared to theory predictions [7, 8].
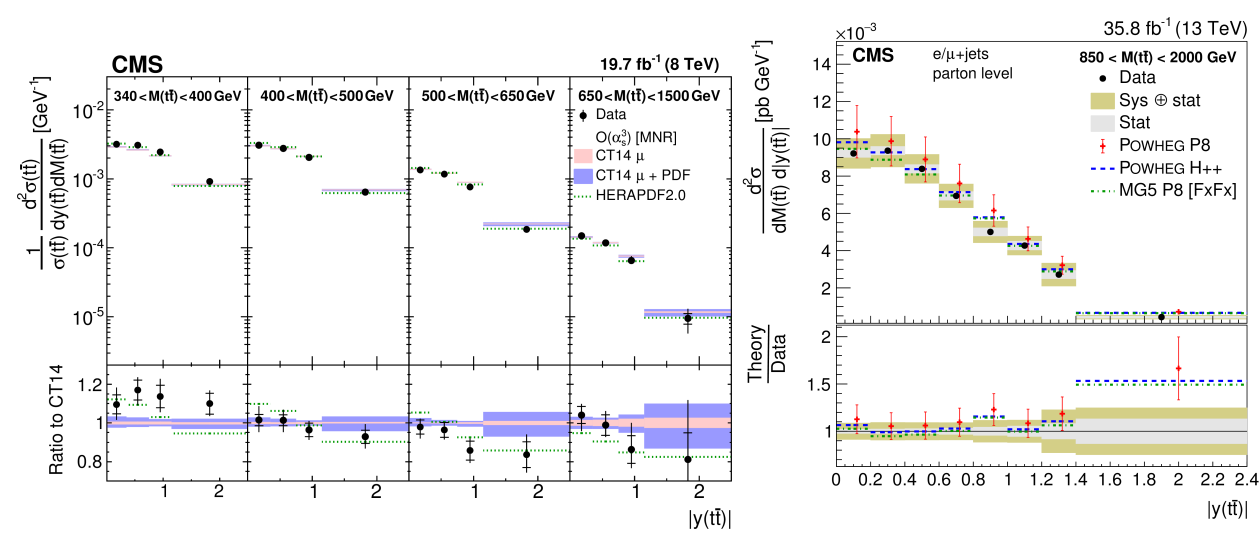

Figure 4: Differential $t \bar{t}$ cross section in all jets channel at 8 and $13 \mathrm{TeV}$ are measured as a function of the leading top quark $p_{T}$ at parton level in the boosted (left) and detector level in the resolved and boosted regime (right). The measurements are compared to theory predictions $[9,6]$.

\section{Differential cross section of t-channel single top quark}

Differential t-channel single top quark cross sections at $13 \mathrm{TeV}$ [10] are measured. Figure 5 shows the measurements as a function of the top quark $p_{T}$. Horizontal ticks on the error bars indicate the statistical uncertainty and vertical bars indicate the total uncertainty per bin. The measurements are compared to theoretical predictions. The first bin of top quark $p_{T}$ has a relatively large uncertainty due to the low acceptance and large sensitivity to the systematic uncertainties.

\section{Conclusions}

Differential cross sections of $t \bar{t}$ and $\mathrm{t}$-channel single top are measured using data collected by CMS. Measurements are performed in dilepton, lepton+jet, and all jet channels at different energies of 8 and $13 \mathrm{TeV}$. The measurements in the boosted regime are included using jet substructure 

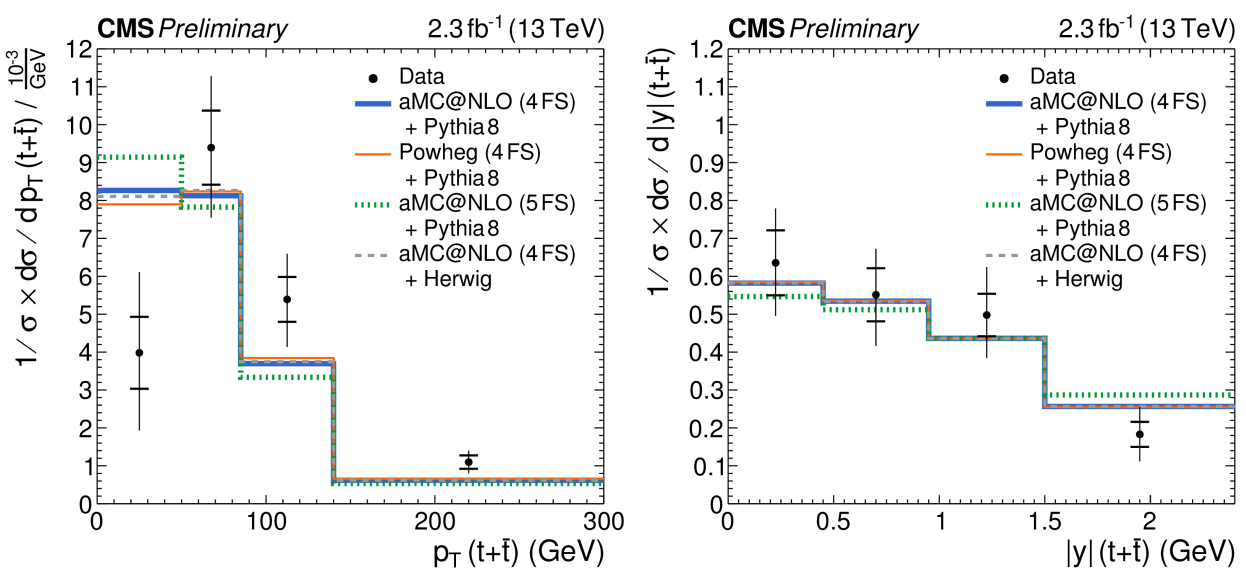

Figure 5: Differential t-channel single top quark cross section at $13 \mathrm{TeV}$ are measured as a function of the top quark $p_{T}$ and rapidity. Horizontal ticks on the error bars indicate the statistical uncertainty and vertical bars indicate the total uncertainty per bin. The measurements are compared to theory predictions [10].

techniques. Also, double-differential cross sections are measured. Differential t-channel single top quark cross sections are measured at $13 \mathrm{TeV}$. In general, the measurements are found to be in good agreement with the predictions within uncertainties.

\section{Acknowledgments}

This study was carried out with financial support of the National Research Foundation of Korea (NRF), funded by the Ministry of Science \& ICT under contract NRF-2008-00460. It was also supported by the Basic Science Research Program through the National Research Foundation of Korea (NRF) funded by the Ministry of Education (NRF-2016R1A6A3A11933762) and by a Korea University Grant.

\section{References}

[1] CMS Collaboration, “The CMS experiment at the CERN LHC”, JINST 3 (2008) S08004

[2] CMS Collaboration, "Measurement of the differential cross section for top quark pair production in pp collisions at 8 TeV”, Eur. Phys. J. C 75 (2015) 542

[3] CMS Collaboration, "Measurement of normalized differential ttbar cross sections in the dilepton channel from pp collisions at 13 TeV", JHEP04 (2018) 60

[4] CMS Collaboration, "Measurement of differential cross sections for top quark pair production using the lepton+jets final state in proton-proton collisions at 13 TeV”, Phys. Rev. D 95 (2017) 092001

[5] CMS Collaboration, "Measurement of differential cross sections for the production of top quark pairs and of additional jets in lepton+jets events from pp collisions at 13 TeV”, Phys. Rev. D 97 (2018) 112003

[6] CMS Collaboration, "Measurement of the integrated and differential $\bar{t} \bar{t}$ production cross sections for high- $p_{\mathrm{T}}$ top quarks in pp collisions at $\sqrt{s}=8 \mathrm{TeV}$ ", Phys. Rev. D 94 (2016) 072002 
[7] CMS Collaboration, "Measurement of the differential $t \bar{t}$ cross section with high- $p_{T}$ top-quark jets in the all-hadronic channel at $8 \mathrm{TeV}$ ", CMS-PAS-TOP-16-013 (2016)

[8] CMS Collaboration, "Measurement of the $t \bar{t}$ production cross section at $13 \mathrm{TeV}$ in the all-jets final state", CMS-PAS-TOP-16-013 (2016)

[9] CMS Collaboration, "Measurement of double-differential cross sections for top quark pair production in pp collisions at $8 \mathrm{TeV}$ and impact on parton distribution functions", EPJC 77 (2017) 459

[10] CMS Collaboration, "Measurement of the differential cross section for t-channel single-top-quark production at $13 \mathrm{TeV}$ ", CMS-PAS-TOP-16-004 (2016) 